\title{
Nuevos paradigmas de la educación universitaria en el Siglo XXI -Reflexión desde la UNPHU y FUDPHU-
}

\section{The New Paradigms of University Education: 21st Century -Reflection from UNPHU and FUDPHU-}

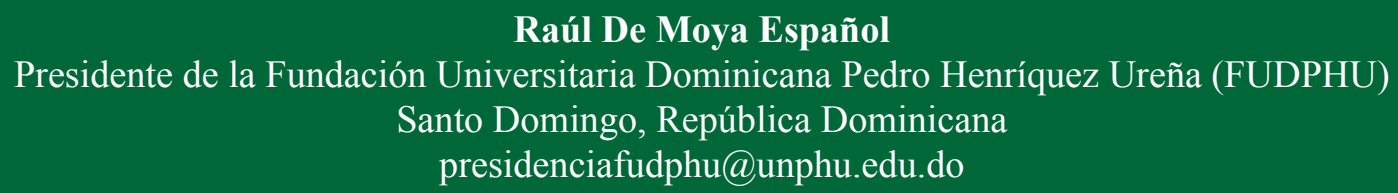

Fecha de recepción: 16 de junio de 2017

Fecha de aceptación: 24 de julio 2017

Favor de citar este artículo de la siguiente forma:

De Moya, R. (2017). Los nuevos paradigmas de la Educación Universitaria: Siglo XXI.

Revista AULA. Vol. 61, Número 1, julio-diciembre 2017. Santo Domingo: Amigo del Hogar

\section{RESUMEN}

Esta reflexión realiza una mirada crítica hacia la Educación del siglo XXI desde la perspectiva de los efectos de los cambios del siglo, la tecnología y su relación con culturas de avance lento expuestas al cambio constante. Analiza, además, la necesidad de detectar las causas de esta última problemática para tomar medidas oportunas y evitar que se pierda el impacto que ejercen las universidades sobre la sociedad y su futuro. Profundiza la mirada, cuestionando la función de las fundaciones modernas en relación a las universidades que patrocinan como son las de sostenerlas, servir de asesores, y hasta de fiscalizadores, evitando a toda costa drenar las arcadas de las instituciones que están dadas a proteger.

\section{Palabras Clave: cambio, cultura, docentes, desarrollo, educación, fundaciones, universidades, siglo XXI, paradigmas}

\begin{abstract}
This reflection takes a critical look at Education in the 21 st century from the perspective of the effects of the changes of the century, technology and its relationship with slow-moving cultures exposed to constant change. It also analyzes the need to detect the causes of the later problem to take timely measures to avoid losing the impact of universities on society and its future. It deepens the view, questioning the function of the modern foundations in relation to the universities that they sponsor as they are to support them, to serve as advisers, and even as auditors, avoiding at all costs to drain the arcades of the institutions that are given to protect.
\end{abstract}

Keywords: Change, culture, teachers, development, education, universities, foundations, technology, 21 st century, paradigms 


\section{Los nuevos paradigmas de la Educación Universitaria siglo XXI -Reflexión desde la UNPHU y FUDPHU-}

Es necesario en las universidades de este siglo una gobernanza con una visión unitaria de sus dos vertientes vitales: la académica pura y la correspondiente a una gerencia contemporánea que vincule la institución con la sociedad y coadyuve con una verdadera visión de futuro donde rijan los principios y valores de una universidad abierta al cambio, orientada al humanismo, la ciencia y la tecnología. Siempre en busca del bienestar de la educación del país. La academia, por su parte, debe mantener una autonomía ordenada en base a normativas claras y consensuadas y cuidar de siempre mantenerse actualizada para ejercer sus funciones sustentada en un aseguramiento de la calidad constante.

Las fundaciones deben ser respetuosas de los aspectos académicos, programas, contenidos, evaluaciones, docencia y demás acciones puras de la academia. Su cogobierno va más en la ayuda a la sostenibilidad financiera y a su vinculación con la sociedad. Ambas acciones deben ser realizadas de manera colaborativa pero con la responsabilidad de parte de la fundación de tener ante quién justificar gastos, inversiones y retribuciones. Se espera de la universidad un uso racional de los recursos y una lógica de sostenibilidad al hacer sus reportes de ejecución y planificar su futuro. De esta forma, apoyándose y haciendo cumplir la misión de cada parte, se logra la fórmula de éxito.

Esta institución se ocupa del logro de las metas de sostenibilidad financiera, aportes a los programas y al desarrollo de vínculos nacionales e internacionales: se vincula con los gobiernos, organismos bilaterales, empresas y otras fundaciones. Este organismo tan impor- tante y sin fines de lucro tiene sus funciones como la universidad tiene otras, sin embargo, se entrelazan y fortalecen.

En esta era de la información, el conocimiento, la ciencia y dinámica tecnológica, el sistema educativo superior dominicano se encuentra en un desarrollo lento, aunque sostenido. La velocidad de su avance aún no ha permitido que surjan de las universidades nacionales las soluciones a la problemática de la sociedad a pesar del interés gubernamental y privado.

Lo mismo sucede con la investigación cuya iniciación plena resulta difícil. Y es que como bien indica Aguerondo (1993a, párr. 4): "Más ciencia y tecnología en nuestra vida cotidiana no ha significado, sin embargo, más ciencia y tecnología en la institución que prepara a las generaciones jóvenes para insertarse en la sociedad, o sea en la escuela". La falta del entrenamiento no solo en el aspecto de investigación pura sino en la gestión de los fondos es parte de las causas de esta circunstancia.

En base a estas mismas limitaciones económicas, lamentablemente, existen instituciones que no cuentan con la tecnología necesaria para los estudiantes y profesores. Esas limitaciones son las que impiden que muchos profesores cuenten con las herramientas que les permitan mantenerse al día tanto en información como en el uso mismo de los instrumentos tecnológicos.

Y así, los nuevos graduandos nativos digitales tienen pocas razones que los motiven a integrarse a la docencia. Hoy, se les incita para que sean emprendedores de sus propios negocios y los beneficios personales de ser docentes en el mundo de la educación superior no 
logran inspirarlos ni competir ventajosamente con esta primera opción. Entonces, persiste el "gap" tecnológico y los profesores, inmigrantes digitales, docentes de otros tiempos siguen luchando por sobrevivir frente a la nueva cultura. Siguen haciendo el mayor esfuerzo para alcanzar la meta tecnológica de la época en que les ha tocado enseñar: la alta tecnología que avanza a pasos agigantados exigiendo una constante conectividad.

Los alumnos, en muchos casos y como consecuencia de lo antes expresado, con frecuencia, son más avanzados que los profesores en lo que a tecnología se refiere y como resultado tienen más acceso a la información. "Los docentes inmigrantes digitales asumen que los estudiantes son los mismos que siempre han sido, y que los mismos métodos que funcionaron para sus docentes con ellos van a funcionar con sus actuales estudiantes. Pero esa asunción ya no es válida”. (Presky, 2001, párr. 14). Los estudiantes de hoy hablan un nuevo lenguaje que los profesores luchan por dominar. Un reto espinoso, pero posible de superar a través del entrenamiento docente y al lograr que la tecnología sea de fácil acceso para los profesores.

Desde otro punto de vista, consideremos ahora, el estudio realizado sobre la "Evolución de la educación superior dominicana y de la extensión universitaria" en relación a la etapa final del siglo XX al siglo XXI y veamos cómo presenta el desarrollo y sus limitantes.

[...] Consolidación del sistema de educación superior y la gestión de la extensión universitaria la cual continúa desarrollándose de forma limitada. El final del siglo XX e inicio del siglo XXI se caracteriza por consolidar el sistema de educación superior dominicano, donde se infiere que las Instituciones de Educación Superior (IES) responden a los señalamientos de la evaluación quinquenal y tratan de fortalecer las debilidades. La educación superior dominicana actual presenta una situación heterogénea protagonizada por una gama de IES. Éste sistema permitió en seis décadas la fundación de 49 IES, algunas de ellas con condiciones débiles para el desarrollo de la gestión de los procesos de docencia, investigación y extensión. Presentando un desequilibrio entre los procesos universitarios y su encargo social, por lo cual se dificulta llevar a cabo actividades de extensión pertinentes, que impacten a la sociedad y permitan que las instituciones del sistema consoliden su identidad para enfrentar los retos de un mundo globalizado. Del estudio de la gestión de extensión universitaria, se desprende que las IES realizan actividades de extensión de forma limitada, y algunas carecen de unidades dedicadas a esta labor como tal. (Goico \& Portuondo, 2013, párr.20)

Visto lo anterior es imperativo que aseguremos la calidad de la educación dominicana, su desarrollo y avance, asunto que se convierte en prioridad con solo ver los resultados del estudio PISA (Programa para la Evaluacion Internacional de Alumnos) en los cuales la República Dominicana ha quedado con un puntaje de 139 del mayor de 436 (OECD, 2015). Y aunque en el estudio no se trata de la educación superior nos da un sabor de lo que podríamos encontrar en este tipo de instituciones, sobre todo en sus niveles básicos.

En realidad, podemos expresar con certeza que hay causas que deben ser determinadas para que los efectos puedan ser superados: currículo, preparación docente, nivel de preparación estudiantil... Es importante que sean determinadas antes de que se pierda para siempre el impacto positivo que ejercen las universidades sobre la sociedad y su futuro. 
Causas, muchas veces provenientes de los efectos del impacto de las economías mundiales sobre los sistemas educativos. Una vez más resulta interesante ver la opinión de Aguerondo (2013b párr. 3) en su trabajo sobre la calidad en la educación: "Bastantes diagnósticos han demostrado empíricamente los problemas de burocratización de la administración, rutinización de las prácticas escolares, obsolescencias de los contenidos curriculares e ineficiencia de los resultados finales".

Sin embargo, y para ser justos, esto no quiere decir que los estudiantes superen en experiencias y conocimientos a sus docentes. $\mathrm{Y}$ es que el conocimiento requiere pasar la información por un juicio crítico, por un proceso de análisis. Es solo así, mediante la capacidad de discernimiento que puede surgir el exquisito filtrado convertido en conocimiento útil y aplicable que permite la toma de decisiones y la solución de problemas. De modo tal, que a pesar de la distancia en destrezas tecnológicas, los profesores siguen siendo los guías indiscutibles del proceso de enseñanza y aprendizaje. Queda claro que las nuevas tecnologías nos permiten ponernos en contacto con la información, pero es la mente humana la que forma, discrimina $\mathrm{y}$ crea nuevos mundos.

Dominar la tecnología sin desarrollar la capacidad de análisis crítico, de comunicación, de discriminación, de reflexionar y de ser capaz de determinar qué conducta tomar ante los dilemas que presenta la vida puede resultar en un vano e infructuoso esfuerzo. Décadas atrás con el advenimiento de los ordenadores surgió la posibilidad de manipular objetos en el computador como uno de los beneficios del sistema. Pudimos copiar textos y cambiarlos de lugar, facilitando el manejo del material escrito, pero algunas personas en poco tiempo le encontraron un uso menos honroso y hoy el "copy-paste" y los plagios arrastran consigo hasta los más serios intentos de nuestras universidades en lograr tesis de grado de valor y transcendencia.

Son tiempos de reflexión sobre lo simple y lo complejo. La dinámica de cambio constante así lo requiere. Ya las facultades no pueden ser conservadoras, ya no pueden verse como estructuras permanentes y como bien expresa Bustamante (2016): [...] "o las universidades se adecuan a los cambios que suceden en el mundo o perderán su razón de ser”. Y en verdad, de no adaptarse, tanto ellas como sus decanos y sus directores quedarán fosilizados en el tiempo. Las universidades deben ser laboratorios de ideas, mundos de creatividad libre donde nuevos conceptos y formas diferentes de accionar florezcan y sean valorados.

La ciencia y la tecnología avanzan a pasos agigantados como sombra que corre a mayor velocidad que el cuerpo al que acompaña. El profesional en formación ve cómo el futuro lo atrapa en pleno presente. Vive con celeridad lo que parecía estar a años luz de distancia. El futuro se hace presente demasiado rápido. Y lo aprendido se convierte en pasado antes de que se afiance.

Anteriormente, las universidades mostraban y analizaban el pasado, vivían el presente y pronosticaban el futuro para aterrizar su ejercicio y vincularlo con sus raíces... Pero, hoy, vivimos en un mundo globalizado donde se van perdiendo las raíces y cada día nos damos cuenta que estamos permeados por culturas extranjeras. Y no cuestionamos si mejores o peores que la nuestra, porque las culturas sencillamente son, pero son valores y contravalores que nos afectan de manera inevitable, aunque reconocemos la opinión de Bellereza, Tavalar y Plazola (2013, párr. 31) cuando concluyen que mientras no desarrollemos una empatía cultural, no podremos 
entender las diferentes maneras de actuar y pensar lo que hace que se complique la operatividad de una organización o el desarrollo de un nuevo negocio en otro país.

Pero, de vuelta al tema que nos ocupa es bueno preguntarse: ¿Estamos educando para el futuro? La pregunta resulta, verdaderamente, retadora. Para poder hacer esto tendremos que afrontar dos aspectos fundamentales: La actualización docente con la incorporación progresiva de nuevos talentos y la revisión profunda de las estructuras organizacionales universitarias acorde a una nueva visión de la enseñanza; no solo de las universidades en sí, sino de todo el sistema universitario nacional, regional y mundial, incluyendo su gobernanza.

Nos referimos a una universidad abierta, es decir, aquella con un sistema flexible, donde se propicie el estudio independiente y se eliminen los obstáculos de ingreso como son: el lugar, edad y trabajo que impedían que cualquier persona que cumpliera con los requisitos de ingreso pudiera optar por un título universitario. Todo esto es posible con la educación virtual a través del sistema de aprendizaje individual distributivo (el estudiante estudia individualmente en su tiempo y espacio, pero se comunica con el mundo) entre otros estilos modernos de aprendizaje como lo define la Universidad de Harvard en el curso Leaders of Learning (Lideres del aprendizaje) que imparte el profesor investigador Richard Elmore (2016) de Harvard edx.

Veamos lo que es educar para el futuro y utilicemos como muestra al Proyecto Tuning. $\mathrm{Y}$ es que estos nuevos tiempos requieren de nuevas medidas como las que se han venido logrando con este importante proyecto que logró que más de 135 universidades Europeas crearan el Espacio Europeo de Educación Superior. Por su lado, Latinoamérica, también, ha jugado un importante papel al crear la versión regional que ha desarrollado titulaciones fácilmente comparables y comprensibles; un alto nivel de convergencia en la educación; desarrollado perfiles profesionales en términos de competencias genéricas y relativas a cada área de estudio; facilitado la transparencia en las estructuras educativas y fomentado la innovación a través de la comunicación de experiencias; la identificación de buenas prácticas; y el desarrollo de los currículos. (Tuning Latinoamérica, 2013). ¡Magnífica forma de abordar el futuro con redes $y$ puentes entre las universidades y otras entidades apropiadas y calificadas para producir convergencia en las áreas de las disciplinas seleccionadas!

Hay que pensar fuera de lo aceptado como patrón para poder innovar ¿Por qué un estudiante dominicano no puede elegir cursar una asignatura de su interés con un profesor que imparte clases en otra universidad del mismo país o del extranjero? ¿Por qué no acreditar este tipo de entrenamiento o de sistemas? Esto complementaría su formación; a la vez que el propio país se beneficiaría de un profesional más completo e integral. ¿No es acaso la formación mediocre la culpable de muchos males de ética profesional e incapacidades que afecta a toda la sociedad?

Es tiempo de vínculos más directos, no solo con otras universidades, el gobierno y sus instituciones de educación superior y la sociedad a la que servimos sino también, de vínculos participativos entre las universidades y las empresas.

"El modelo para la transformación de la universidad tradicional en emprendedora tiene su origen a mediados y finales del siglo $\mathrm{XX}$ en Estados Unidos y se encuentra aplicado en 
el Instituto de Tecnología de Massachusetts (MIT) o la Universidad de Berkeley en California, que fueron pioneras en la explotación comercial de los resultados de la investigación”. (Del Castillo \& Reyes, 2015, párr. 58).

Hoy, universidades como la de Cambridge tienen laboratorios de investigación, departamentos de emprendimiento y ofrecen a sus estudiantes la comercialización de cualquiera de los descubrimientos. Y así, presentan con orgullo "Negocios desde la universidad de Cambridge". Los fondos provenientes del Cambridge Enterprise Funds proponen a los alumnos e inversionistas la oportunidad de invertir en el desarrollo de descubrimientos científicos y tecnológicos realizados en la universidad. Por su lado, Cambridge Enterprise, subsidiaria de transferencia de tecnología de la universidad, es responsable de los arreglos de comercialización para los descubrimientos de la Universidad y cuenta con la asesoría financiera de Parkwalk Advisors. (Cambridge, 2107, párr. 1)

Muchos son los modelos de universidadempresa, pero solo mencionaremos varios para los fines de este trabajo: El modelo de universidad-empresa de Sábato, el modelo triple hélice y el estatalista son referentes que sirven como base a estas aseveraciones. El modelo Sábato plantea un triángulo de interacciones entre el gobierno, la empresa y la universidad. El Modelo de la Triple Hélice facilita la comprensión de la relación universidad-empresa. A través de los conceptos capitalización del conocimiento universitario y universidad emprendedora señala un camino en dicha relación, observándose la importancia de la transformación de las instituciones universitarias en emprendedoras para dicho vínculo. (Del Castillo \& Reyes, 2015, párr. 60). Los modelos estatalistas, por su parte vinculan el estado, la industria y la universidad al modelo tradicional de "no interferencia" donde cada parte actúa de forma independiente.

Como ven, la nueva universidad es una universidad emprendedora con nueva visión de trabajo conjunto que permea las estructuras, otrora rígidas, y permite que aun en facultades y estructuras internas los "académicos" se acompañen de profesionales que puedan dar ideas de sostenibilidad financiera y gestionar su consecución.

Lo ideal es que las empresas sean parte de las universidades y que las universidades sean parte de las empresas. No se puede seguir educando en una burbuja de cristal donde lo que pasa dentro no, necesariamente, está vinculado con lo que pasa afuera. La universidad debe ser ejemplo, por ella misma, de lo que enseña. Es por tanto fundamental que revisemos cómo estamos estructurados para funcionar y cómo debemos adecuarnos para hacerlo dentro del nuevo contexto en que se desarrolla nuestra sociedad y el mundo.

Hecha esta salvedad, penetraremos otros ámbitos con preguntas puntuales: ¿Acaso debemos seguir formando para que un estudiante pase cinco años aprendiendo cosas que al terminar sus estudios, en ocasiones, ya resultan caducas? La respuesta es obvia: debemos educar basados en planes estratégicos claros, precisos y con indicadores críticos que contengan las competencias que puedan ser aplicadas durante su formación y que garanticen un aprendizaje útil y aplicable para el resto de su vida. Entonces, hay que enseñar al estudiante dónde buscar la información o el conocimiento que le ocupa; guiarlo en el arte de investigar para que pueda discriminar lo que es importante y lo que no. Hay que lograr desarrollar la competencia básica de aprender a aprender para que lo pueda hacer de manera autónoma para el resto de su vida evitando que 
el conocimiento caduque. Por otro lado, las universidades a su vez, deben mantenerse a la vanguardia con bibliotecas con recursos de información, tecnología de punta y con espacios de reflexión donde se analicen y discutan los asuntos vitales del mundo, de la región y del propio país.

¿Cuántos cambios y giros de camino tendrá que realizar un profesional desde el inicio al termino de sus estudios?; ¿cómo cambiará su vida si tomamos en cuenta que muchas de las carreras que estudian los jóvenes de hoy pueden en esta época de la tecnología, sencillamente desaparecer?; ¿que harán cuando la sociedad, como sucede en tantos casos, no le brinde la ocasión de ejercer o que las oportunidades los dirijan hacia otras áreas? ¿Qué estamos haciendo al respecto? No parece que nos estemos preparando para ese momento. Para que entendamos con mayor facilidad este fenómeno que sucede frente a nuestros ojos, veamos algunos ejemplos que hemos vivido de segunda mano y que Chuet-Missé de EDEconomía Digital especifica:

[...] los agentes de viajes van siendo reemplazados por las páginas de reservas de vuelos y alojamientos por Internet. La banca sigue reduciendo personal y cerrando oficinas: sólo en España, proyecta despedir a 21.000 empleados en tres años. La digitalización de las operaciones, el uso de los cajeros automáticos y las transferencias por móvil reducen el papel del empleado bancario a un área marginal, limitada a la atención personalizada a las personas que no se encuentran cómodas con la tecnología o a los grandes privados.

(Chuet-Missé, 2017, párr. 5)

En verdad, serían interminables y sumamente interesantes las mesas de reflexión sobre la necesidad de una visión analítica de la sociedad actual y las proyecciones futuras de la educación... Difícil y extensas reflexiones cuando tendríamos que reconocer que se sigue educando como el siglo XX, inmersos en plena profundidad del siglo XXI.

Los protagonistas de esta nueva era son aquellos con la capacidad de mutar, formando parte de la transformación, acompañando y sobre todo siendo los líderes de los cambios, que aunque evidentes frente a un análisis sagaz, son muchas veces invisibles a simple vista porque se expresan de manera soterrada.

Hay otras preguntas de interés que surgen de este tipo de reflexiones: ¿Quiénes asumen las investigaciones en las sociedades? En las del mundo desarrollado, de las universidades y las empresas de otros ámbitos, solo nos llegan como tendencias, ideas y olas de pensamientos... Ahí está la historia para demostrarlo. Y por solo poner un ejemplo: las enfermedades tropicales raramente son afrontadas para su minimización o eliminación. El trópico es visto como subdesarrollo, nuestras universidades no cuentan dentro de las que investigan porque las grandes farmacéuticas de los países desarrollados son las que se han adueñado de estos renglones prioritarios.

Hay que cambiar, tenemos que crear centros de investigación dedicados a los males que afectan nuestras sociedades, no solo en el ámbito de la salud sino también el de las humanidades y ciencias sociales, al igual que en los campos de la economía, medioambiente, vivienda, en fin..., áreas de desarrollo de las cuales nuestras regiones adolecen. Para ello, son las escuelas de graduados las puertas a la investigación; son las maestrías y doctorados los instrumentos. Las escuelas de postgrado deben estar vigilantes frente a las ofertas que transformarán la realidad del subdesarrollo y la pobreza en una 
realidad nueva y no debe centrarse, solamente, en ofertas económicamente rentables, pero que no impactan la sociedad de manera positiva al punto de permitirle superar sus grandes dificultades.

Y así, sobrevenimos al tema de los recursos humanos: la posición geográfica, el clima social y político, y su condición tropical, pueden atraer un personal docente valioso que muchas veces es subutilizado en otros países; maestros científicos e investigadores que con frecuencia son retirados justo cuando están en pleno dominio del arte de su ciencia. En nuestro país estamos dispuestos a aprovechar estas mentes brillantes y convertir su retiro en la oportunidad de captar personal formado que puede entrenar a los locales.

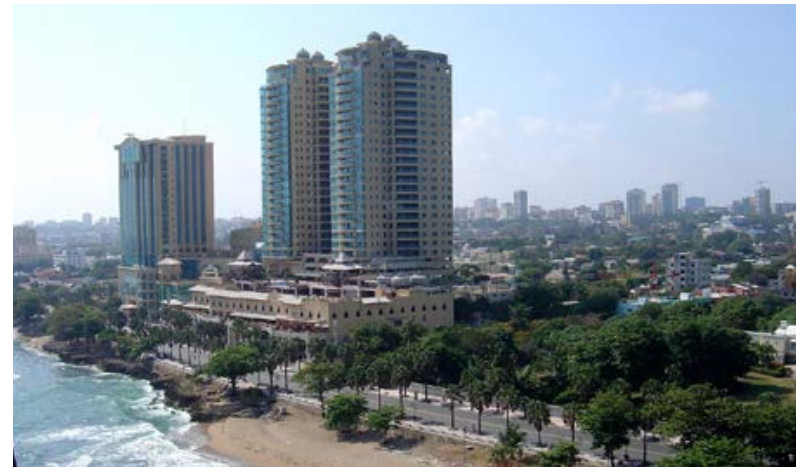

Imagen 1. Ciudad de Santo Domingo, República Dominicana.

Ahora, de manera puntual nos adherimos a la necesidad de crear un Centro Regional de Escuelas de Graduados donde participen las universidades acreditadas apoyadas en sus fortalezas e incentivadas por el ministerio pertinente. Otros países del área han tenido protagonismo en tiempos diferentes... México, Chile, Argentina, Cuba y Puerto Rico. Y ahora es nuestro tiempo. Tiempo de crear la "universidad abierta" para una vinculación real y efectiva. Laboratorio de ideas e investigaciones enlazadas con universidades y empresas destacadas, con visión de futuro y estructuradas de forma pertinente para responder a las necesidades de la sociedad. Visión de futuro como la planteada por la Fundación Universitaria de CAFAM y el Ministerio de Educación Nacional (MEN):

La visión de futuro nos invita a actuar de forma proactiva ideando ese mañana, desde nuestros valores, principios, actitudes y fortalezas. Supone liderar y soñar el destino donde queremos estar. Esa claridad es la que nos mueve y nos da la fuerza de voluntad suficiente para empezar a generar los cambios para el logro del futuro deseado. Es una actitud emprendedora necesaria para sacar adelante los sueños personales, familiares, educativos y por supuesto empresariales. Tener una visión requiere de un punto de referencia de éxito que nos permite cambiar nuestros paradigmas y ser más competitivos para superar los desafíos del siglo XXI; ayuda a generar confianza en mí mismo y el querer colaborar con otros en aras de construir un espacio de relaciones donde las diferencias individuales se convierten en una fuente de oportunidades.

(Unicafam, s/f, párr. 2)

Algunas personas podrían entender que el rol de las fundaciones no es necesario ni importante para el desarrollo de la educación, pero ya hemos visto la incidencia positiva de ciertas fundaciones en el curso de este trabajo.

El punto es importante y aunque pueda lucir una extrapolación, no lo es. Pero veamos ahora a vuelo de pájaro, pero con desplazamiento certero, las fundaciones universitarias y su efecto. Se trata de hacer notar que tanto los miembros del Consejo Universitario como la gobernanza de la Fundación se deben nutrir y apoyar para el logro de la excelencia académica y de la competitividad deseada con otras instituciones latinoamericanas. 
Acompañar, auspiciar, apoyar y fiscalizar son las funciones principales de las fundaciones universitarias sin fines de lucro. Lo primero es que los miembros de una fundación universitaria tienen que entender y hacer suya la misión y visión de la organización universitaria, y sobre todo de una organización de estos tiempos, asunto a todas luces retador. Fiscalizar pudiera lucir una palabra fuerte, pero se refiere a velar que la universidad maneje bien, no solo el proceso educativo, sino los fondos de la institución en vías de cuidar las sostenibilidad de la misma. No se trata de una forma inquisitiva y limitante de supervisar, tampoco de intervenir o interferir en el libre fluir del trabajo o de censurar el proceso educativo: se trata de velar, cuidar, vigilar y asegurar la buena educación y administración.

Hoy día una entidad que esté "fiscalizada", deja claro que es una organización que practica la transparencia, no sólo que la predica, por tanto quien "fiscaliza" o promueve una educación en valores debe enseñar con el ejemplo. Esto claramente hace que aún las entidades auspiciadoras o fiscalizadoras o que ejercen cogobierno, aún sean entidades sin fines de lucro como las fundaciones, deben ser un libro abierto y ser objeto ellas mismas de auditorías y fiscalización, para de esta forma estar certificadas por la sociedad como tal.

Es necesario que los miembros de una fundación universitaria estén al tanto del acontecer académico y administrativo, de las nuevas tendencias, de lo que conviene a la universidad para pautar un futuro de seguridad y bienestar para la institución. Los miembros de una fundación universitaria, asimismo, requieren de actualización en cómo apoyar, auspiciar y fiscalizar. Muchas veces se trae al escenario de las fundaciones visiones que son muy válidas dentro del ámbito del ejercicio de quien las trae, pero no así dentro del organismo, la fundación debe ser capaz de separar las conveniencias personales de las institucionales y actuar en consecuencia a favor de la universidad.

Recordemos para los fines de lugar que hoy día existe el concepto de "universidad empresa" y que una fundación dedicada a la Educación debe evitar a toda costa ser un huésped comensal de la universidad que patrocina. $\mathrm{Su}$ función principal es darle vida y ayudar a la universidad a su consolidación y desarro1lo. Deben y tienen que ser autosuficientes a través de un modelo de gestión ejemplar; entonces, serán capaces de mostrar un patrón a seguir. Los recursos de las fundaciones y su sostenibilidad deben ser ejercidos de forma tal que su operación esté al servicio de la universidad como centro de formación, investigación y extensión en una gerencia académica pertinente y regulada.

Pongamos como ejemplo la Universidad de Sacramento: a través de su fundación University Enterprises, Inc. (UEI) y basada en el concepto universidad-empresa realiza una actividad de inversión para fortalecer el campus al proporcionar beneficios que no pueden lograrse con los fondos derivados de la matrícula o de fondos estatales:

"La actividad de inversión de UEI fortalece el campus al proporcionar beneficios que no pueden lograrse con los fondos estatales. Ejemplos: incluyen compras en edificios, subvenciones de investigación federales y recursos vitales como la librería Hornet, los servicios de comedor y los Lofts de Upper Eastside. En los últimos años, el apoyo de UEI al estado de Sacramento ha generado millones de dólares para iniciativas de enseñanza y aprendizaje, así como un mejor espacio para los estudiantes. Estos servicios y funciones esenciales cuentan con un acuerdo operativo con la universidad. 
UEI es una corporación sin fines de lucro gobernada por un consejo de administración de conformidad con los códigos apropiados del Estado de California y las directivas de política de la Junta de Síndicos y la administración del campus". (University Sacramento, Inc., 2017, párr. 3).

Veamos otros ejemplos: La junta directiva (Board of Trustees) de la Universidad de Cornell tiene control total sobre los asuntos de la universidad incluyendo sus facultades. En cuanto a la de Harvard, la función principal de la junta es alentar a la Universidad a mantener los más altos estándares alcanzables como centro de aprendizaje. La Junta ejerce una amplia influencia sobre las direcciones estratégicas de la Universidad, ya que proporciona asesoramiento esencial para el liderazgo de la Universidad en cuanto a prioridades y planes. Tiene el poder de dar su consentimiento a ciertas acciones como la elección de miembros de la Corporación, y dirige el proceso de visitación mediante el cual se revisa periódicamente una amplia gama de escuelas y departamentos de Harvard.

Viendo hacia Latinoamérica, presentamos el ejemplo de la Universidad Simón Bolívar que basada en el concepto "universidad-empresa" creó la Fundación Funindes-USB donde el concepto de cooperación o vinculación se refiere a convenios que pueden establecerse entre unidades académicas y empresariales.

Se habla indistintamente de la relación universidad con el sector externo, empresa, sector productivo, industria. Se entiende por sector externo, el entorno global con el cual interactúa la universidad, mientras que la industria-empresa es toda una entidad productiva, pequeña, mediana o grande, bien sea por su capital o por el número de personas que laboren en ellas, pudiendo ser públicas o priva- das, nacionales o transnacionales. Las actuales formas de cooperación con las empresas son múltiples y variadas, a saber: prestación de servicios académicos, cuando la universidad brinda ayuda de consultoría y asistencia técnica a través del trabajo de sus docentes más capacitados; investigación y desarrollo experimental y proyectos de innovación tecnológica, entre muchos otros servicios que ofrece a las empresas y por los cuales recibe una remuneración. Estos recursos pueden ser utilizados para aminorar o eliminar las causas que afectan la calidad de la educación como son recursos didácticos, mejoría de la estructura física, equipos tecnológicos, en fin...

De la relación "universidad-empresa" surgen relaciones, recursos económicos y humanos que permiten mejorar la calidad de la educación superior en todos los aspectos y posibilita superar obstáculos y satisfacer las necesidades internas en todos los ámbitos: educación continuada; adquisición de recursos didácticos y tecnológicos; la vida laboral de los docentes y administrativos, mejora de la infraestructura, y del plan de compensaciones, entre otros.

Libretti (1999, párr.25), por su lado, nos habla de los muchos aportes y beneficios que brindan las fundaciones universitarias, entre ellos: dar soporte a los investigadores en el proceso de negociación de contratos de investigación; búsqueda de financiamiento en fuentes gubernamentales; estrategias de protección industrial y redacción de patentes; seguimiento de los proyectos contratados entre universidad y empresa...

La fundación será el mejor testigo de la creación de alianzas. Esta idea puede generar apoyo de empresas ya que dentro de lo que establece la ley, se puede deducir hasta el 5\% del pago a impuestos internos cuando realizan donaciones para fines educativos. Estas empresas 
acompañarían a la fundación no solo con el aporte, sino como un patrono vigilante del uso de esos recursos.

Bien constituida en su forma operativa las fundaciones de este tipo pueden y deben abrirse a la sociedad para que en comisiones específicas de personalidades y empresas participen en proyectos puntuales de mutuo interés para beneficio de las instituciones de educación superior. Serán canales de apoyo a las universidades a quienes auspicien, generadoras de proyectos, de programas, de oportunidades y recursos, además, de ser celosos vigilantes del fiel cumplimiento de sus presupuestos y metas estratégicas.

Es importante propiciar el posicionamiento social de la universidad dentro del sector a que pertenece. La fundación no debe competir con las funciones de la universidad. Si bien, debe ser parte indispensable de su equilibrado y sostenido desarrollo. El éxito de las universidades es el éxito de sus fundaciones y así deben celebrarlo.

La gestión financiera de la universidad debe ser aprobada mediante un presupuesto y vigilada mediante un sistema de auditoría de gestión y resultado. Las fundaciones no deben aplicar el presupuesto universitario, sino aprobarlo o sugerir los cambios requeridos por el bien de las academias, por lo que no deben ser quienes firmen erogaciones haciéndose así principales responsables de lo que luego auditarán. El conflicto de intereses debe ser evitado a toda costa y las reglamentaciones de doble auditoriainterna y externa suman valor en la calidad del proceso, entre otras medidas de interés.

Las fundaciones universitarias deben ser un ejemplo ético de la gerencia del siglo XXI. En países en vías de desarrollo debemos tomar en cuenta estudios como los de Vidal (2006, párr. 3) sobre las entidades sin ánimo de lucro donde afirma que "A mayor riqueza económica: se supone que el bienestar es un bien normal, por consiguiente, cuanto mayor sea el nivel de riqueza del país o de la región, mayor será el nivel de demanda de bienes relacionados con el bienestar social: cultura, servicios sociales, consumo responsable, sanidad". Pero, después de la salud, ¿acaso hay un bien social más importante que la educación?.

Asimismo, valoramos y propiciamos la participación de voluntarios basada en el deseo de aportar y de servir para el desarrollo sostenido del sistema educativo nacional. La Fundación Universitaria Dominicana Pedro Henríquez Ureña, Inc. (FUDPHU) cuida de todos estos detalles. Desde su concepción ha sido una institución democrática que elige a doce miembros como Junta de Administración de la Fundación; doce iguales para garantizar que haya una participación democrática y plural, solo se eligen entre ellos los cargos operativos para poder funcionar adecuadamente y dar responsabilidades dentro de iguales. Este criterio debe reforzarse y auspiciarse al fomentar la participación y no la exclusión, y trabajar con consensos sin castigar el disenso, si queremos una sociedad democrática incluyente y participativa.

En consecuencia, y después de todo lo antes dicho, la calidad de la educación debe ser asegurada y para ello debemos hacer un estudio donde se determine el estatus general de la educación superior en nuestro país, buscando determinar las causas de la situación local. Además, se deberá planificar salidas creativas frente a la desaparición de gran cantidad de trabajos dado los notables y continuos avances tecnológicos. La educación continua y la movilidad de los docentes serán acciones que, sin lugar a dudas, mejorarán la educación en República Dominicana. Por su lado, las fundaciones 
universitarias que se entregan al desarrollo de la educación juegan un papel vital en la evolución de los pueblos y quienes las componen no solo tienen la responsabilidad inmensa de reenfocarse, constantemente, sino que deben estar conscientes de que dejan su impronta grabada en la historia de la trasformación de los pueblos y que con su accionar dan la mejor lección: la de educar con el ejemplo.

\section{Referencias}

Aguerondo, I. (1993a). El Nuevo

Paradigma de la Educación para el siglo. Madrid. Organización de

Estados Iberoamericanos para la Ciencia y la Cultura para la educación la ciencia y la cultura (OIC). Recuperado de URL: http://www.oei.es/historico/administra-cion/aguerrondo.htm

Aguerondo, I. (1993b). La calidad de la educación: Ejes para su definición y evaluación. Tomado el 13 de julio de URL: http://campus-oei.org/calidad/ aguerrondo.htm

Bustamante, L. (2016). Cambios y retos de la educación universitaria: entrevista con Luis Bustamente. Recuperado de URL: https://youtu.be/4IFDuKMTxCs. Guatemala. Universidad Francisco Marroquín, New media UFM.

Berrelleza, M., Talavar, C. \& Plazola, M. (2013). Identificar las diferencias culturales que afectan en el desarrollo de los negocios internacionales. Recuperado de URL: https://www.ride.org.mx/ index.php/ RIDE/article/view/72/307

Chuet-Missé, J. (2017). Cuáles son los trabajos que desaparecerán en 30 años. EDeconomiaDigital. Recupera- do de URL: http:// www.economiadigital. es/politica-y-so-ciedad/cuales-sonlos-trabajos-que-desa-parece ran-en-30-anos_188941_102.html

Cornell University (s.f.). Board of Trustees. Recuperado de URL:https://trustees. cornell.edu/Pages/welco-me.aspx

Del Castillo, L, \& Reyes, S. (2015). Los modelos de relacion universidad empresa. Revista: Caribeña de Ciencias Sociales. ISSN: 2254-7630. Recuperado de URL: http://www.eu-med.net/rev/ caribe/2015/07/universidad-empresa. html

Elmore, R. (2016). Leaders of Learning. [Lideres del aprendizaje]. Massachusetts. Harvard edx. Recuperado de URL: https://www.edx.org/course/lea ders-learning-harvardx-gse2x-1

Goico, J. \& Portuondo, R. (2013). Evolución de la educación superior dominicana y de la extensión universitaria. UCE Ciencia. Revista de postgrado. Vol. 1 (3), 2013. Recuperado de URL: uceciencia.edu.do/index. php/ OJS/article/download/70/64

Harvard Alumni (2017). Harvard Governing Board. [Gobernanza de Harvard]. Recuperado de URL: https:// alumni. harvard.edu/about-haa/board/ cu-rrent-members

Libretti, V. (1999). Un modelo de Unidad de vinculación universidad-empresa. Funindes-USB. Universidad Simón Bolívar. Recuperado de UL: http:// www.revistaespacios.com/ a99v20n01/40992001.html\#formas 
OECD (2015). Singapur encabeza la última encuesta PISA sobre educación que realiza la OCDE a escala internacional. Recuperado de URL: http://www.oecd.org/pisa/ singapur-encabeza-la-ultima-encuesta-pisa-sobre-educación-que-realiza-laoc-de-a-escala-internacional.htm

Prensky, M.(2001). Digital Natives, Digital Immigrants. On the Horizon Vol. 9 No. 5, pp. 1-6

Tuning Latinoamérica (2013). Tuning Latinoamérica: Antedecedentes y objetivos. Recuperado de URL: http://tuning.unideusto.org/tunin-gal/index php?option $=$ content\&task=-view\&id=171\&Itemid=199

University of Cambridge (s.f). Business activity at the University of Cambridge and information for businesses. [Actividad de negocios en la Universidad de Cambridge e información para negocios]. Cambridge: Reino Unido. Recuperado de URL: http://www.cam.ac.uk/ for-businesses

University Enterprises, Inc (s.f.). University Enterprises, Inc., a non-profit auxiliary organization of California State University, Sacramento, creates and manages programs and services that enhance the University services that enhance the University's educational mission. California. Recuperado de URL: http://www.enterprises. csus.edu/about/

Unicafam (s.f.). Visión de futuro. Recuperado de URL: http://chec.unicafam. edu.co/index.php/category/instituciones-educativas/

Vidal, I. (2006). Las entidades sin ánimo de lucro en el siglo XXI. Recuperado de URL: http://les.practi-casdeclase. web-node.es/200000152-37d2638ccb/ Empre-sas_sin_\%20animo_lucro.pdf

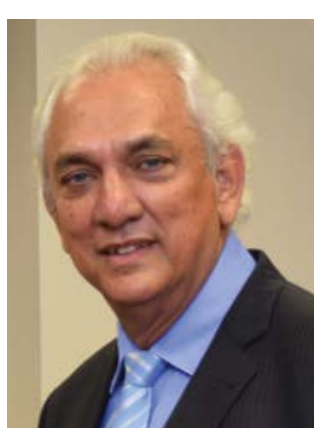

\section{Raúl De Moya Español}

Es presidente de la Fundación Universitaria Dominicana Pedro Henríquez Ureña (FUDPHU), arquitecto y urbanista graduado de la Universidad Nacional Pedro Henríquez Ureña; Máster de Gestión de Proyectos otorgado por la Université de Québec en Outaouais de Canadá. Postgrados en: Estructura para Arquitectos de la UASD; Arquitectura y Clima, CODIA; Preparación y Evaluación de Proyectos; Arquitectura Sismo Resistente, CETEC; Planificación, Programación y Control de Proyectos, UNICEF; Arquitectura Cubana; Gerencia de Proyectos de Construcción, UCSD. Sus investigaciones han sido publicadas bajo los títulos: "Una estrategia para el enfoque del Diseño Arquitectónico"; "Evaluación de la Producción Informal de Viviendas en República Dominicana" (bajo los auspicios de las Naciones Unidas); "Metodología para la Elaboración de Proyecto de Grado". Ha sido merecedor de los siguientes premios:1er lugar en la 1ra Bienal de Arquitectura Dominicana categoría "Teoría y Crítica de la Arquitectura"; 1er. lugar de la 2da Bienal de Arquitectura Dominicana, categoría "Proyecto"; Mención honorífica en la 4ta Bienal de Arquitectura Dominicana en la categoría "Proyecto" con "La vivienda progresiva"; 1994 Mención Honorífica en la 5ta Bienal de Arquitectura Dominicana categoría "Obra" con "Centro Parroquial Buen Pastor". Se ha desempeñado como docente en las siguientes instituciones: UNPHU, UCIFAS, UASD, PUCMM. Ha sido presidente (1998 - 2000) de la Sociedad de Arquitectos. Consultor local del BID para la evaluación sectorial de viviendas para la República Dominicana y Miembro del Consejo de Directores del CONAU. 